\title{
Mixed Near-field and Far-field Sources Localization via Second-order Statistics
}

\author{
Zhong-xi XIA ${ }^{1, a}$, Xiao-fei ZHANG ${ }^{1,2,3}$, Wei-tao LIU ${ }^{1}$, Qian-lin $\mathrm{CHENG}^{1}$, and \\ Dong-lin YANG ${ }^{1}$
}

1. College of Electronic and Information Engineering, Nanjing University of Aeronautics and Astronautics, Nanjing, 210016, People's Republic of China

2. State Key Laboratory of Millimeter Waves, Southeast University, Nanjing, China, 210096.

3. National Mobile Communications Research Laboratory, Southeast University, Nanjing, China, 210096.

axiazhongxi@nuaa.edu.cn

Keywords: Mixed near-field and far-field, localization, Second-order Statistics.

\begin{abstract}
This paper proposes an algorithm for mixed near-field and far-field sources localization, using the trilinear decomposition (PARAFAC) model via second-order statistics of the received signal. We construct two second order statistical matrices of the received signal and use PARAFAC model to obtain the parameters of all sources, then according to the definition of distance of near-field source, that we can correctly distinguish the near-field and far-field sources, and we can get the exact parameters estimation of all the sources. This method does not need eigenvalue decomposition of the covariance matrix of the received signal, and does not need to airspace traverse search, so it greatly reduces the computational complexity and automatically matches the parameters, avoiding the parameter matching process. MATLAB simulation results show that this is an effective parameter estimation algorithm for mixed near-field and far-field sources localization.
\end{abstract}

\section{Introduction}

Source localization can be classified into far-field source DOA estimation and near-field source localization, according to the range between the sources and the array. In both cases, the wave-fronts of incoming signals are different completely, so that they have different signal models. In the far-field source localization, the wave-front of the incoming signal is assumed to be a plane wave propagating in space [1], so each source is parameterized by only the Direction-Of-Arrival (DOA). Many classical algorithms have been developed to solve this problem, such as the maximum likelihood (ML) method [2-6], the MUSIC method [7-9], and the ESPRIT method [10-12]. But in the near-field sources localization, the signal wave-front is spherical, and both the DOAs and ranges are needed to localize near-field sources. There are a lot of algorithms that have been raised for near-field source localization, for example, the maximum likelihood (ML) method [13], the 2D-music method [14, 15], the second-order statistics method [16, 17], the fourth-order cumulants method [18] and so on.

In many engineering applications, the mixing field contains near-field sources and far-field sources, that is the near-field sources and far-field sources will appear at the same time. At this point, the algorithm is required not only need to distinguish between the near-field and far-field sources, but also to achieve accurate parameters estimation of the sources. There are many algorithms that have been proposed for mixed near-field and far-field sources localization [19-22], but if the algorithm that is necessary to decompose the eigenvalues of the received signal covariance matrix, or need to airspace traverse search, that is too complicated. For the cumulant-based algorithm can avoid spatial traversing the airspace search, thus it greatly reduce the computational complexity. Therefore, we propose an algorithm based on the second-order statistic matrix, this algorithm does not need to decompose eigenvalue of the received signal matrix. It does not need the aerial search process, so the calculation is greatly reduced, and in this paper we use three-linear least squares method, so the 
estimated parameters are automatically paired, after MATLAB simulation experiments verify that this is an effective algorithm for mixed near-field and far-field sources localization.

\section{Data model}

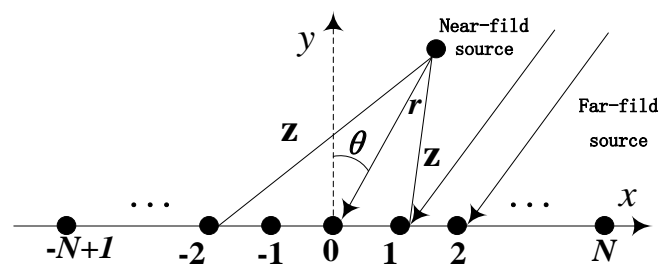

Fig. 1 ULA for mixed source localization

We consider that there are 3 narrowband and independent mixed near-field and far-field sources ( $K=3, K_{n f}=2, K_{f f}=1$ ), radiated to the ULA, which contains $2 \mathrm{~N}$ equally spaced omnidirectional sensors, as shown in Fig 1. the intersection of the array is selected as the reference point of the phase. The signals received by the $(i, 0)$ th and $(0, i)$ th sensor in the subarray can be expressed as follows [23],

$$
x_{m}(t)=\sum_{k=1}^{K} s_{k} e^{j \tau_{m k}}+n_{m}(t),-N \leq m \leq N
$$

Where $S_{k}$ is the $k$-th $(k=1,2, \ldots, K)$ source arrives at the $m$-th element received and demodulated baseband signal, $n_{m}$ is the additive white noise on the elements. $\tau_{m k}$ is the signal sent by the $k$-th source arrives at the phase difference between the $m$-th element and the arrival of the reference element. For the range of near-field sources $r_{k} \in\left[0.62\left(D^{3} / \lambda_{k}\right)^{1 / 2}\left(2 D^{2} / \lambda_{k}\right)\right]$, According to Fresnel approximation, $\tau_{m k} \approx \gamma_{k} m+\phi_{k} m^{2}$, Where $\gamma_{k}=-2 \pi \frac{d \sin \theta_{k}}{\lambda_{k}}, \phi_{k}=\pi \frac{d^{2}}{\lambda_{k} r_{k}} \cos ^{2} \theta_{k}, \lambda_{k}$ is wavelength of the $k$-th signal, $\theta_{k} \in[-\pi / 2, \pi / 2]$, It is can be seen from the above equations, When $r_{k}$ tends to infinity, $\phi_{k}$ tends to 0 . So the above formula can be approximated as, $x_{m}(t) \approx \sum_{k=1}^{K} S_{k} e^{j\left(\gamma_{k} m+\varphi_{k} m^{2}\right)}+n_{m}(t)$, written in the form of matrix,

$$
\mathbf{X}(\mathrm{t})=\mathbf{A S}(\mathrm{t})+\mathbf{N}(\mathrm{t})
$$

While the matrix of receive signal is $\mathbf{X}=\left[x_{-N}(t), \ldots, x_{0}(t), \ldots, x_{N}(t)\right]^{T}$, the direction matrix is $\mathbf{A}=\left[a_{\left(\theta_{1}, r_{1}\right)}, \ldots, a_{\left(\theta_{k}, r_{k}\right)}\right]$, the matrix of source signal is $\mathbf{S}=\left[s_{1}(t), \ldots, s_{k}(t)\right]^{T}$, the matrix of noise is $\mathbf{N}=\left[n_{-N}(t), \ldots, n_{0}(t), \ldots, n_{N}(t)\right]^{T}$, The superscript $T$ indicates transpose.

Throughout the rest of the paper, the following hypotheses are assumed to hold.

(1) The sources are statistically mutually independent of narrow-band stationary processes with non-zero kurtosis.

(2) The noise is zero-mean Gaussian signal and independent of the signals, its variance is $\sigma^{2}$.

(3) The source parameters are different from each other, $\phi_{i} \neq \phi_{j}, \quad r_{i} \neq r_{j}$.

(4) The different elements are isotropic, there is no channel inconsistency, mutual coupling and other factors, gain of the space is set to 1 .

(5) In order to avoid phase blur, the distance between the elements must be met $d \leq \min \left(\lambda_{k} / 4\right)$. 
(6) The range of near-field source $r_{k} \in\left[0.62\left(D^{3} / \lambda_{k}\right)^{1 / 2}\left(2 D^{2} / \lambda_{k}\right)\right]$, the far-field source $r_{k} \rightarrow \infty$, $\phi_{k} \rightarrow 0$.

\section{Algorithm Description}

3.1 Define two second-order statistics matrices. Similar to [24], we defined the second-order statistics matrix $\mathbf{R}_{1}, \mathbf{R}_{2}$, for different sensor and time lags, the (m,n)-th element of which has the following form,

$$
\begin{aligned}
& \mathbf{R}_{\mathbf{1}}(m, n)=E\left\{x_{m-n+1}(k) x_{m-n}^{*}(k)\right\}=\sum_{l=1}^{L} r_{s l} e^{j\left(\gamma_{l}+\phi_{l}\right)} e^{j 2(m-n) \phi_{l}} \\
& \mathbf{R}_{2}(m, n)=E\left\{x_{n-m}(k) x_{n-m+1}^{*}(k)\right\}=\sum_{l=1}^{L} r_{s l} e^{-j\left(\gamma_{l}+\phi_{l}\right)} e^{j 2(m-n) \phi_{l}} .
\end{aligned}
$$

Where $1 \leq m, n \leq N, r_{s k}=E\left\{x_{k}(\mathrm{t}) x^{*}{ }_{k}(\mathrm{t})\right\}$ is the power of the l-th source, which is assumed to be nonzero.

Here we define $\boldsymbol{\Lambda}=\operatorname{diag}\left\{\begin{array}{lll}e^{j \phi_{1}} & \cdots & e^{j \phi_{1}}\end{array}\right\}, \boldsymbol{\Omega}=\operatorname{diag}\left\{e^{j \gamma_{1}} \cdots e^{j \gamma_{1}}\right\}, \boldsymbol{\Gamma}=\operatorname{diag}\left\{r_{s 1}, r_{s 2}, \cdots, r_{s L}\right\}$. So the Eq. 3 and Eq. 4 can be written as $\mathbf{R}_{\mathbf{1}}=\mathbf{A} \boldsymbol{\Gamma} \mathbf{\Omega} \mathbf{\Lambda} \mathbf{A}^{H} \in \mathbf{R}^{M \times M}, \mathbf{R}_{\mathbf{2}}=\mathbf{A} \boldsymbol{\Gamma} \boldsymbol{\Omega}^{-1} \boldsymbol{\Lambda}^{-1} \mathbf{A}^{H} \in \mathbf{R}^{M \times M}$, Where $\mathbf{A}=\left[a_{x 1}, a_{x 2}, \ldots, a_{x K}\right]$, it is an equivalent direction matrix [25].Because of the assumptions $i \neq j, \quad \phi_{x i} \neq \phi_{x j}, \quad \gamma_{z i} \neq \gamma_{z j}$ and $r_{i} \neq r_{j}$, So $\mathbf{A}$ is an equivalent direction matrix with rank $\mathrm{K}$.

3.2 Parameter estimation. As one of the methods for fitting PARAFAC model, trilinear alternating least square (TALS) approach [26], Here, we can construct model as follow,

$$
\left[\begin{array}{l}
\mathbf{R}_{1} \\
\mathbf{R}_{2}
\end{array}\right]=\left[\begin{array}{l}
\underline{\mathbf{X}}(:,:, 1) \\
\underline{\mathbf{X}}(:,:, 2)
\end{array}\right]=\mathbf{A}\left[\begin{array}{c}
\Gamma \mathbf{\Omega} \boldsymbol{\Lambda} \\
\Gamma \mathbf{\Omega}^{-1} \boldsymbol{\Lambda}^{-1}
\end{array}\right] \mathbf{A}^{H}+\mathbf{N}
$$

The superscript $H$ denotes the Hermitian transpose, we define $\mathbf{C}=\left[(\boldsymbol{\Gamma} \boldsymbol{\Omega} \boldsymbol{\Lambda})^{T}\left(\boldsymbol{\Gamma} \boldsymbol{\Omega}^{-1} \boldsymbol{\Lambda}^{-1}\right)^{T}\right]$, and $\mathbf{B}=\mathbf{A}^{H}$, the Eq. 5 can be written as follows,

$$
\mathbf{Z}=\left[\begin{array}{l}
\underline{\mathbf{X}}(:,:, 1) \\
\underline{\mathbf{X}}(:,:, 2)
\end{array}\right]=\left[\mathbf{Z}_{1}^{T} \cdots \mathbf{Z}^{T}{ }_{N}\right]=\mathbf{A}\left[D_{1}^{T}(\mathbf{C}) \cdots D_{N}^{T}(\mathbf{C})\right] \mathbf{B}+\mathbf{N}=(\mathbf{C} \odot \mathbf{A}) \mathbf{B}+\mathbf{N} .
$$

Similarly, using the symmetry of the PARAFAC model, we can construct following slice matrices, we can get $\mathbf{X}=\left(\mathbf{A} \odot \mathbf{B}^{T}\right) \mathbf{C}^{T}+\mathbf{N}, \quad \mathbf{Y}=\left(\mathbf{B}^{T} \odot \mathbf{C}\right) \mathbf{A}^{T}+\mathbf{N}$. So, we can get $\hat{\mathbf{A}}^{T}=\left[\mathbf{C} D^{T}{ }_{1}\left(\mathbf{B}^{T}\right) \cdots \mathbf{C} D^{T}{ }_{N}\left(\mathbf{B}^{T}\right)\right]^{+}\left[\mathbf{Y}_{1} \cdots \mathbf{Y}_{N}\right]^{T}, \hat{\mathbf{B}}$, and $\hat{\mathbf{C}}^{T}$, Where $(\bullet)^{+}$Expressed fake inverse, through the above formulas, such an alternating projections-type procedure is iterated for all subsets of parameters until the convergence is achieved. We can get the estimate of the parameters $\hat{\mathbf{A}}, \hat{\mathbf{B}}$ and $\hat{\mathbf{C}}$. Then we can get, $\hat{\phi}_{k}=\frac{1}{2(N-1)} \sum_{i=1}^{N-1}\left\{\angle \cdot\left[\frac{\hat{\mathbf{A}}(i+1,:)}{\hat{\mathbf{A}}(i,:)}\right]\right\}, \hat{\gamma}_{k}=\frac{1}{2} \angle \bullet\left[\frac{\mathbf{C}(1,:) e^{-2 j \hat{\phi}_{k}}}{\mathbf{C}(2,:)}\right]$, So the parameter estimation of the sources can be obtained, $\hat{\theta}_{k}=-\arcsin \left(\frac{\hat{\gamma}_{k} \lambda_{k}}{2 \pi d}\right), \hat{r}_{k}=\frac{\pi d^{2}}{\lambda_{k} \phi_{k}} \cos ^{2} \hat{\theta}_{k}$. 
3.3 Parameter estimation. We can distinguish near-field sources and far-field sources from the parameter estimation of $\hat{r}_{k}$. If $\hat{r}_{k} \in\left[0.62\left(D^{3} / \lambda_{k}\right)^{1 / 2}\left(2 D^{2} / \lambda_{k}\right)\right]$, the $k$-th sources are near-field sources, or they are far-field sources, while $1 \leq k \leq K$.

$$
r_{k}=\frac{\pi d^{2}}{\lambda_{k} \phi_{k}} \cos ^{2} \hat{\theta}_{k} \begin{cases}\in\left[0.62\left(D^{3} / \lambda_{k}\right)^{1 / 2}\left(2 D^{2} / \lambda_{k}\right)\right], & \text { the } k \text {-th source are near-field source } \\ >\left(2 D^{2} / \lambda_{k}\right), & \text { the } k \text {-th source are far-field source }\end{cases}
$$

3.4 Algorithm steps. We summarize the algorithm steps presented in this paper as follows,

Step1. Define the two second-order statistics matrix $\mathbf{R}_{1}$ and $\mathbf{R}_{2}$.

Step2. Use the PARAFAC model, get the estimate of the parameters $\hat{\mathbf{A}}, \hat{\mathbf{B}}$ and $\hat{\mathbf{C}}$.

Step3. Get the parameters $\hat{\gamma}_{k}$ and $\hat{\phi}_{k}$, then get the parameters estimation of sources $\left(\hat{\theta}_{k}, \hat{r}_{k}\right)$.

Step4. Distinguish near-field sources and far-field sources.

\section{Simulation Results}

Consider there are 2 near-field and 1 far-field narrowband independent sources impinging upon a ULA consists 14 elements, which spacing $d=(\lambda / 4)$.Each Monte Carlo simulation experiment runs 500 times independently. The performance of the algorithm is measured by the root mean square error (RMSE) of the estimated parameters, and it is shown in the follow formula, for example,

$$
\operatorname{RMSE}_{\theta}=\sqrt{\frac{1}{M} \sum_{m=1}^{M}\left(\hat{\theta}_{m}-\theta\right)^{2}}
$$

Simulation 1. Consider there are 3 sources located at $\left\{5^{\circ} 0.2 \lambda\right\},\left\{\begin{array}{ll}10^{\circ} & 0.6 \lambda\end{array}\right\},\left\{20^{\circ}+\infty\right\}$, The Fig. 2 depicts the scatter plot of the proposed algorithm for 1000 Monte Carlo simulations when the number of elements in array $M=14$, snapshots $J=300, S N R=20 \mathrm{~dB}$. In order to be able to show the angle information of far-field source, the distance of far-field source defined as 1.

Simulation 2. Consider there are three sources located at $\left\{\theta_{1}=5^{\circ}, r_{1}=0.2 \lambda\right\},\left\{\theta_{2}=15^{\circ}\right.$, $\left.r_{2}=0.4 \lambda\right\},\left\{\theta_{3}=30^{\circ}, r_{3}=\infty\right\}$, while $\mathrm{M}=14$, From Fig. 3, Fig. 4 and Fig. 5 ,Analysis these figures, we can see that, with the increase in number of snapshots and SNR, the accuracy of parameters estimation both in far-field and near-field are also improving.

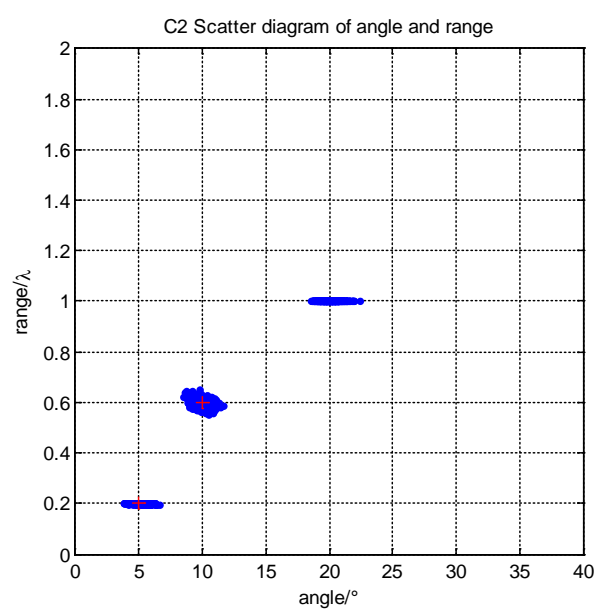

Figure 2. scatter diagram of angle and range

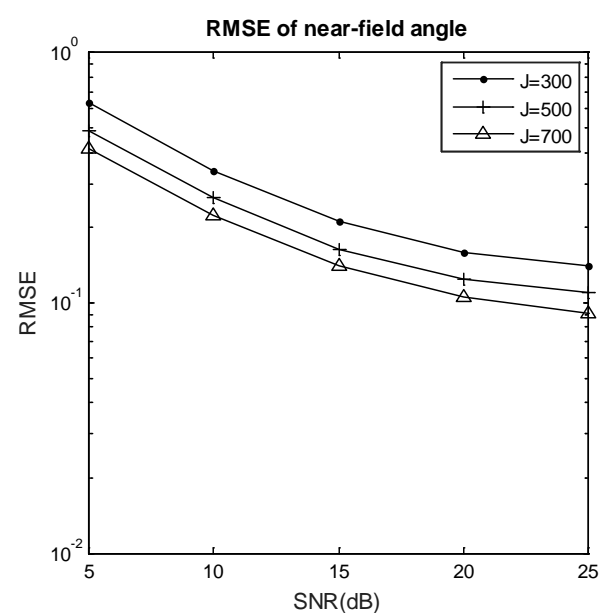

Fig. 3 RMSE of azimuth angle of near-field source 


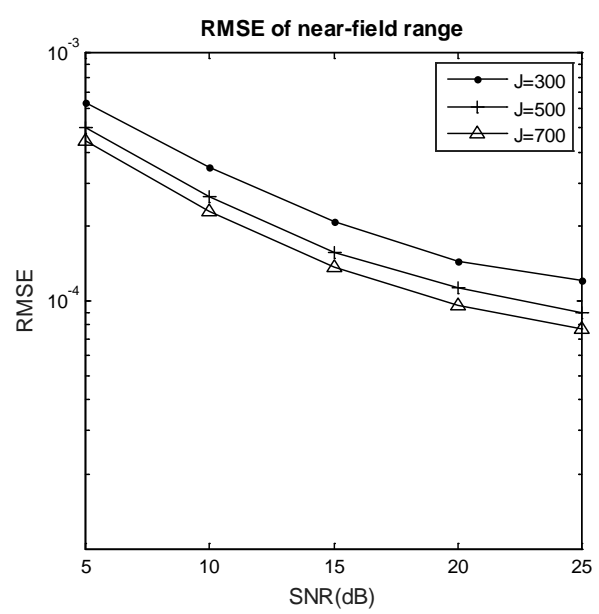

Fig. 4 RMSE of range of near-field source

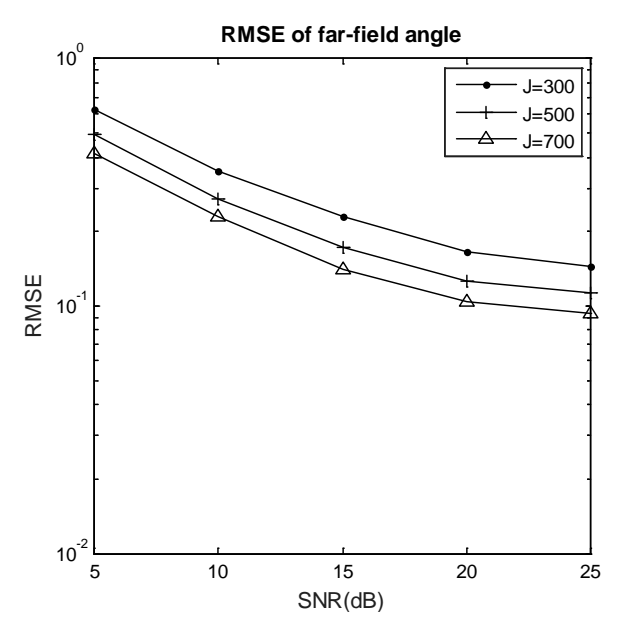

Fig. 5 RMSE of azimuth angle of far-field source

\section{Conclusion}

In this paper, we propose a method for mixed near-field and far-field sources localization, which uses second-order statistics of the received signal, this algorithm does not need to decompose eigenvalue of the received signal matrix. It does not need the aerial search process, so the calculation is greatly reduced. This algorithm can effectively distinguish the near field and far field signal, and the parameters of the source can be estimated directly. After MATLAB simulation experiments verify that this is an effective algorithm for mixed near-field and far-field sources localization.

\section{References}

[1] Krim H, Viberg M. Two decades of array signal processing research:the parametric approach [J].IEEE Signal Processing Magazine, 1996, 13(4):67-94.

[2] Stoica P, Nehorai A. MUSIC, maximum likelihood, and Cramer-Rao bound [J]. IEEE. Transactions on Acoustics Speech \& Signal Processing, 1989, 37(5):720-741.

[3] Stoica P, Nehorai A. Performance study of conditional and unconditional direction-of-arrival estimation [J]. IEEE Trans.on ASSP, 1990, 38(10):1783-1795.

[4] Tsakalides P, Nikias C L. Maximum likelihood localization of sources in noise modeled as a stable process [J]. IEEE Transactions on Signal Processing, 1995, 43(11):2700-2713.

[5] Ye H, Degroat R D. Maximum likelihood DOA estimation and asymptotic Cramer-Rao bounds for additive unknown colored noise [J]. Signal Processing IEEE Transactions on, 1995, 43(4):938-949.

[6] Pesavento M, Gershman A B. Maximum-likelihood direction-of-arrival estimation in the presence of unknown nonuniform noise [J]. IEEE Transactions on Signal Processing, 2001, 49(7):1310-1324.

[7] Schmidt R O. Multiple Emitter Location and Signal Parameter Estimation [J]. IEEE Transactions on Antennas \& Propagation, 1986, 34(3):276-280.

[8] Zoltowski M D, Kautz G M, Silverstein S D. Beamspace Root-MUSIC [J]. IEEE Transactionson Signal Processing, 1993, 41(1):344-364.

[9] Rao B D., Hari K V S. Weighted subspace methods and spatial smoothing: analysis and comparison [J]. IEEE Transactions on Signal Processing, 1993, 41(2):788-803. 
[10]Roy R, Kailath T. ESPRIT-estimation of signal parameters via rotational invariance techniques [J].IEEE Transactions on Acoustics Speech \& Signal Processing, 1989, 37(7):984-995.

[11] Swindlehurst A L., Ottersten B., Roy R., et al. Multiple invariance ESPRIT [J]. Signal Processing IEEE Transactions on, 1992, 40(4):867-881.

[12] Mathews C P, Zoltowski M D. Eigenstructure techniques for 2-D angle estimation with uniform circular array [J]. IEEE Transactions on Signal Processing, 1994, 42(9):2395-2407

[13]Swindlehurst A L, Kailath T. Passive direction-of-arrival and range estimation for near-field sources [C] The Workshop on Spectrum Estimation \& Modeling. IEEE, 1988:123-128.

[14]Huang Y D, Barkat M. Near-field multiple source localization by passive sensor array [J]. IEEE Transactions on Antennas \& Propagation, 1991, 39(7):968-975.

[15]Jeffers R, Bell K L, Van Trees H L. Broadband passive range estimation using music[C] IEEE International Conference on Acoustics. 2002: III-2921 - III-2924.

[16]Abed-Meraim K, Hua Y, Belouchrani A. Second-Order Near-Field Source Localization: Algorithm And Performance Analysis [C] Circuits, Systems and Computers, 1977. Conference Record. 1977 11th Asilomar Conference on. 1996:723-727 vol.1.

[17]Abed-Meraim K, Hua Y. 3-D near field source localization using second order statistics [J]. 1997, 2:1307-1311 vol.2.

[18]Challa R N, Shamsunder S. Passive near-field localization of multiple non-Gaussian sources in 3-D using cumulants 1 [J]. Signal Processing, 1998, 65(1):39-53.

[19]Wang Kai, Wang Ling, Shang Jing-Rui, Mixed Near-Field and Far-Field Source Localization Based on Uniform Linear Array Partition IEEE Sensors Journal ( Volume: 16, Issue: 22, Nov.15, 2016 ) Page(s):8083 - 8090

[20]Liu, Guohong; Sun, Xiaoying Efficient Method of Passive Localization for Mixed Far-Field and Near-Field Sources IEEE Antennas and Wireless Propagation Letters ( Volume: 12 ) Page(s): 902 - 90516 July 2013

[21]J.-J. Jiang, F.-J. Duan, and J. Chen, Three-dimensional localization algorithm for mixed near-field and far-field sources based on ESPRIT and MUSIC method, Progress In Electromagnetics Research, Vol. 136, 435-456, 2013

[22] Huang Y D, Barkat M. Near-field multiple source localization by passive sensor array [J]. IEEE Transactions on Antennas \& Propagation, 1991, 39(7): 968-975.

[23]Liang, Junli; Liu, Ding Passive Localization of Mixed Near-Field and Far-Field Sources Using Two-stage MUSIC Algorithm IEEE Transactions on Signal Processing ( Volume: 58, Issue: 1, Jan. 2010 ) Page(s): 108-120

[24]Abed-Meraim K, Hua Y. 3-D near field source localization using second order statistics [J]. 1997, 2:1307-1311 vol.2.

[25]Chen. Jian-feng, Zhu Xiao-long, and Zhang Xian-da. A new algorithm for joint range-DOA-frequency estimation of near-field sources [J]. EURASIP Journal on Applied Signal Processing, 2004, (3): 386-392.

[26]Liang J, Yang S, Zhang J. A New Near-Field Source Localization Algorithm without Pairing Parameters[C] Sensor Array and Multichannel Processing, 2006. Fourth IEEE Workshop on.2006:162-165. 